\title{
Study of Serum Intestinal Alkaline Phosphatase in Rosacea
}

\author{
Andreea Merticariu' ${ }^{1}$, Luminița Marinescu², Călin Giurcăneanu ${ }^{3,4}$
}

'Sf. Pantelimon Clinical Emergency Hospital, Bucharest, Romania

${ }^{2} \mathrm{CMI}$ Marinescu Luminita, Bucharest, Romania

${ }^{3}$ Elias Clinical EmergencyHospital, Bucharest, Romania

${ }^{4}$ Carol Davila University of Medicine and Pharmacy, Bucharest, Romania
Corresponding author:

Andreea Merticariu, MD

Sf. Pantelimon Clinical Emergency

Hospital, Bucharest, Romania

E-mail: andreeamerticariu@yahoo.com

\section{ABSTRACT}

Background: Rosacea is a chronic inflammatory dermatosis, frequently associated to various gastrointestinal disorders. Recent studies suggest that intestinal alkaline phosphatase, an enzyme that guards intestinal homeostasis might be involved in the pathogenesis of rosacea.

Objective: The objective of this study is to investigate serum intestinal alkaline phosphatase - (IAP) enzymatic activity levels in rosacea patients and correlate them with different clinical forms.

Methods: Twenty six rosacea patients and twenty three age - and sex-matched healthy controls were included in our study. The severity of rosacea was assessed according to the National Rosacea Society classification system. The levels of serum IAP enzymatic activity were measured in all individuals.

Results: There was no statistically significant difference between IAP enzymatic activity levels in the patient and control group, but we demonstrated a significant difference $(p<0.05)$ when comparing patients with papulopustular rosacea to healthy controls and also when comparing patients younger than 40 years to matched controls younger than 40 years. Conclusion: Our results suggest that increasing IAP enzymatic activity especially in papulopustular rosacea by special diet or by IAP supplementation might be beneficial in controlling this disease.

Key words: rosacea, intestinal alkaline phosphatase, age, papulopustular, erythematotelangiectatic

\section{INTRODUCTION}

Rosacea is a common chronic inflammatory facial dermatosis. Although in general rosacea affects young adults in their thirties to fifties with phototype II or III sun sensitive skin any person regardless of skintype or age can develop flushing with diffuse erythema, telangiectasia anda papulopustular eruption, namely the clinical signs specific to rosacea. In the medical literature, rosacea lesions have been reported even in children or in individuals of African descent. The prevalence of the disease in Europe is highly variable; in some countries upto one person in five presents rosacea. It affects women more than men, but the most severe cases appear in males (1). According to the grading system of 
rosacea, published by the National Rosacea Society in The United States of America in 2002, rosacea has four distinct clinical forms: erythematotelangiectatic (RET), papulopustular (RPP), phymatous and ocular (2). Telangiectasia, diffuse erythema and local burning sensation prevail in RET; inflammation with oedema, papules and pinpoint pustules are the hallmark in PPR, while in phymatous rosacea hyperplasia and hypertrophy of sebaceous glands on the nose, chin or less often ears are noticeable. In ocular rosacea patients have mainly ocular signs and symptoms, such as conjunctivitis, blepharitis, conjunctival telangiectasia, dryness or light irritation. Different types of lesions can be present simultaneously in one patient, but the lesion that clinically dominates is important for classifying the disease. Usually, in the evolution of the disease some of the existing clinical features accentuate graduall yor new ones emerge. The latest clinical classifications include uncommon variants of rosacea: granulomatous rosacea, neurogenic rosacea $(3,4)$.

Despite the fact that so many people suffer from rosacea, studies proving that the impact rosacea has on the patient quality of life is similar to psoriasis (5), and that ample research has been done in all aspects of rosacea, scientists still did not succeed to clearly establish the etiology of this condition. Several etiopathogenic hypotheses regarding either local cutaneous anomalies or various infectious microorganisms have been proposed. Presently, it is postulated that vasculature and neuroimmune local dysregulations, dermal matrix degeneration, excessive oxidative stress due to high levels of reactive oxygen species, and abnormally high levels of antimicrobial peptides such as cathelicidins expressed in the skin all play central roles in rosacea development (6). Various studies also proved that pathogens such as Demodex mites, Bacillus oleronius or Helicobacter pylori might be important in initializing the cascade of events that lead to cutaneous inflammation, the pathogenic basis of rosacea $(7,8)$.

It appears that there is a strong link between the skin and the gut microbiota. Dysregulation of the bacterial population resident in the stomach or in the small intestine and certain infectious agents are responsible for gastrointestinal conditions that are described as possible comorbidities for rosacea: chronic gastritis, ulcerative colitis or Crohn's disease (some scientists imply that a not yet identified infectious microorganism might be the cause for these inflammatory bowel diseases) $(9,10)$. In addition rosacea flare-ups can be triggered by certain types of food or beverages, some of which also modulate the activity of intestinal alkaline phosphatase (IAP) (11). IAP is an enzyme localized in the brush border of the intestine with several protective functions. It has complex roles in maintaining gastrointestinal homeostasis and in blocking the inflammatory response triggered by pathogenic bacteria. IAP is involved in detoxification of drugs, heavy metals or foods, in regulating the intestinal surface $\mathrm{pH}$ and the passage of vasoactive peptides released into the intestinal lumen, through the intestinal brush border into the blood stream (12). Hormones such as gastrin, vasoactive intestinal peptide (VIP) and secretin have potent vasodilator properties and are known rosacea triggers. In IAP functional disturbances, dilators have free pathway into the blood and determine worsening of rosacea symptoms (11).

Recent studies showed that IAP might be the missing link to the influence some gastrointestinal conditions or dietary factors have on rosacea development and evolution (11). Therefore we conducted the present study to investigate this possible relationship between rosacea and IAP by evaluating IAP enzymatic activity in rosacea patients.

\section{METHODES}

We recruited anumber of 49 human subjects for our experiment ( 26 rosacea patients and 23 healthy matched controls). Rosacea was diagnosed based on clinical signs and symptoms. We divided our patient group in 2 subgroups, in accordance with the form of rosacea: erythematotelangiectatic rosacea (RET) subgroup (15 patients) and papulopustular rosacea (RPP) subgroup (11 patients). None of the individuals had any systemic treatment or gastrointestinal symptom for the last two months previous to sample collection. Each included subject signed a written informed consent.

In order to evaluate IAP enzymatic activity, a 10 $\mathrm{ml}$ fasting blood sample was collected from each person within our study population. Each sample was immediately centrifuged at $1500 \mathrm{rpm}$, followed by electrophoresis and analysis of enzymatic activity in separate fractions. The results were expressed as $\mu \mathrm{mol} / \mathrm{s}$ land after ward converted in IU/L (commonly used value), using the standard conversion factor.

The statistical methodology used F-test for differences in variances, t-test for differences in means and histograms.

\section{RESULTS}

Our patient group included 17 women with an age range of 21-75 years (mean age 39.58) and 9 men with 
an age range of 19-69 years (mean age 53.22). Sex ratio was approximately $2: 1$. The two mean ages correlate with patients'general behaviour: women seek medical examination at the onset of their disease while men ask medical advice in later, more severe stages. The control group was comparable in terms of sex (17 women, 7 men) and age (women age range 24-68 years, mean age 41.64; men age range $24-66$ years, mean age 47.42 ) to the patient group.

All individuals had total alkaline phosphatase alkaline enzymatic activity within normal reference range (30-120 IU/L). Values for intestinal alkaline phosphatase enzymatic activity did not exceed $15 \%$ of the total alkaline phosphatase value (as considered normal by the laboratory performing the analysis) (13), except one higher value obtained in one control who was referred to gastroenterology. The above mentioned subject was excluded from our study, therefore 48 individuals were observed for this study.

In the rosacea group IAP activity values were normal in relation to total $A P$ and varied from a minimum of $0.00 \mathrm{IU} / \mathrm{L}$ to a maximum of $11.38 \mathrm{IU} / \mathrm{L}$ (mean value $2.1 \mathrm{IU} / \mathrm{L})$. In the control group values varied from a minimum of $0.0 \mathrm{IU} / \mathrm{L}$ to a maximum of $14.97 \mathrm{IU} / \mathrm{L}$ (mean value $3.78 \mathrm{IU} / \mathrm{L}$ ) (table 1, fig. 1).

After we rejected the hypothesis that the variances of the two samples are equal (F-test, $p=0.03$ ), we tested for differences in the means of the patient and control samples. We found no statistically significant differences between IAP levels in the patient ( $M=3.78$; $S D=4.76)$ and control groups $(M=2.1 ; S D=3.26)$ :
Table 1. Statistical characteristics of IAP values in rosacea group and in control group

\begin{tabular}{lcc}
\hline & Rosacea patients & Controls \\
\hline Total individuals & 26 & 22 \\
\hline Mean & $2.10 \mathrm{IU} / \mathrm{L}$ & $3.78 \mathrm{IU} / \mathrm{L}$ \\
\hline Median & $0.00 \mathrm{IU} / \mathrm{L}$ & $2.99 \mathrm{IU} / \mathrm{L}$ \\
\hline Std. deviation & $3.26 \mathrm{IU} / \mathrm{L}$ & $4.76 \mathrm{IU} / \mathrm{L}$ \\
\hline Minimum & $0.00 \mathrm{IU} / \mathrm{L}$ & $0.00 \mathrm{IU} / \mathrm{L}$ \\
\hline Maximum & $11.38 \mathrm{IU} / \mathrm{L}$ & $14.97 \mathrm{IU} / \mathrm{L}$ \\
\hline
\end{tabular}

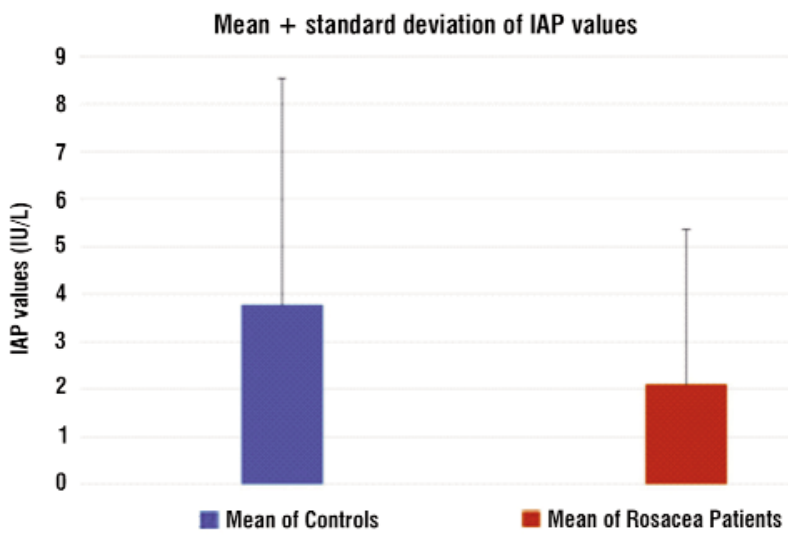

Figure 1 - Mean values of the Controls and Rosacea patients subsamples with one standard deviation bar

$t(36)=1.40, p=0.085$ for one-tail test. However, as the combined histogram from fig. 2 shows, it appears that lower values are more frequent in patients than in controls.

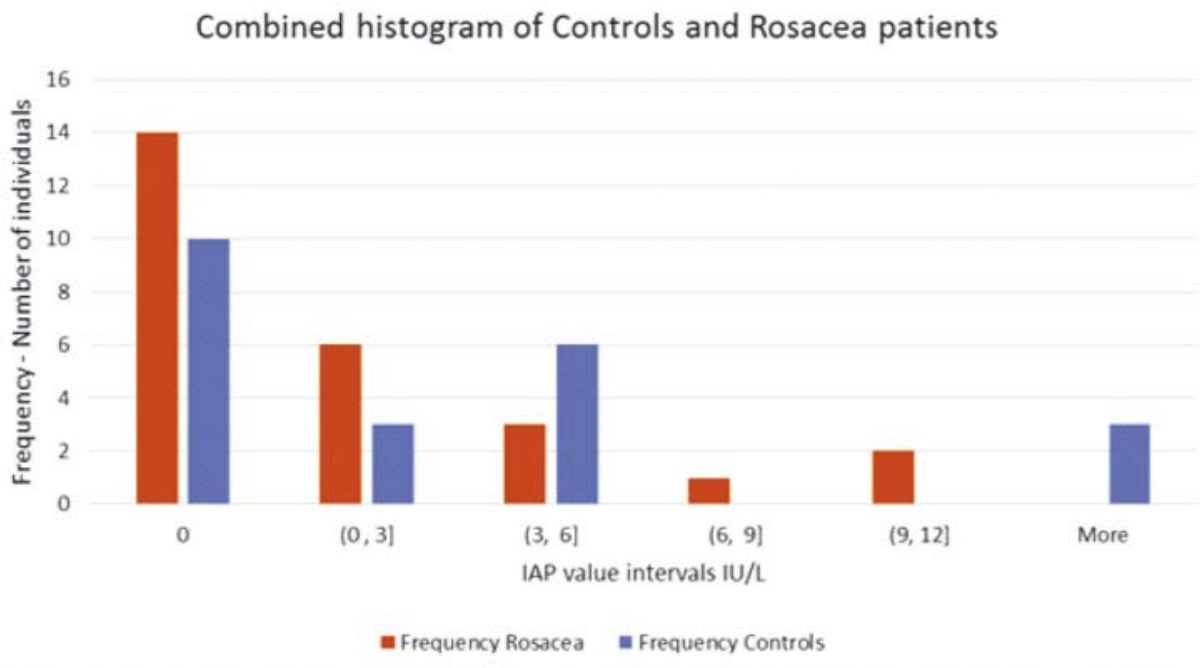

Figure 2 - Frequency of IAP values in patients versus matched controls 
The t-test methodology was repeated in the RET (15 patients) and RPP (11 patients) subgroups. The difference was not statistically significant when the RET subgroup was compared with the controls. In the case of the RPP subgroup, we found evidence for different means with the t-test $t(31)=2.05, p=0.024$. As confirmed by the histograms, the difference between IAP values from controls is less clear in RET patients, but in the case of the RPP subgroup we notice that patients more often have smaller IAP values (fig. 3).

IAP levels were not affected by gender in either patient or control group. Then we investigated whether the age could play a significant role in the distribution of IAP values. The scatter plot of IAP levels against age (fig. 4) shows that there appear to be a positive correlation in the case of patients and a negative one in the case of controls.
We detected a significant difference, $\mathrm{t}(13)=1.85$, $p$-value $=0.043$ for one-tail t-test, assuming unequal variances, between IAP levels in rosacea patients younger than 40 years versus matched controls younger than 40 years (fig. 5). In this patient group we notice a higher distribution of 0.0 IU/L IAP activity value and of small values of IAP, IAP activity being significantly lower in rosacea patients. The 40 years threshold was chosen because in scientific literature in majority of patients this disease debuts after the age of 30 and up to 50 years (14) and because in our study population median age was close to 40 (43 years) I. Thus, the age of 40 could be a relevant threshold, because it assures that our study can find a reasonable sample size of patients while being as close as possible to the age interval that is characteristic for rosacea, i.e., after 30 years.
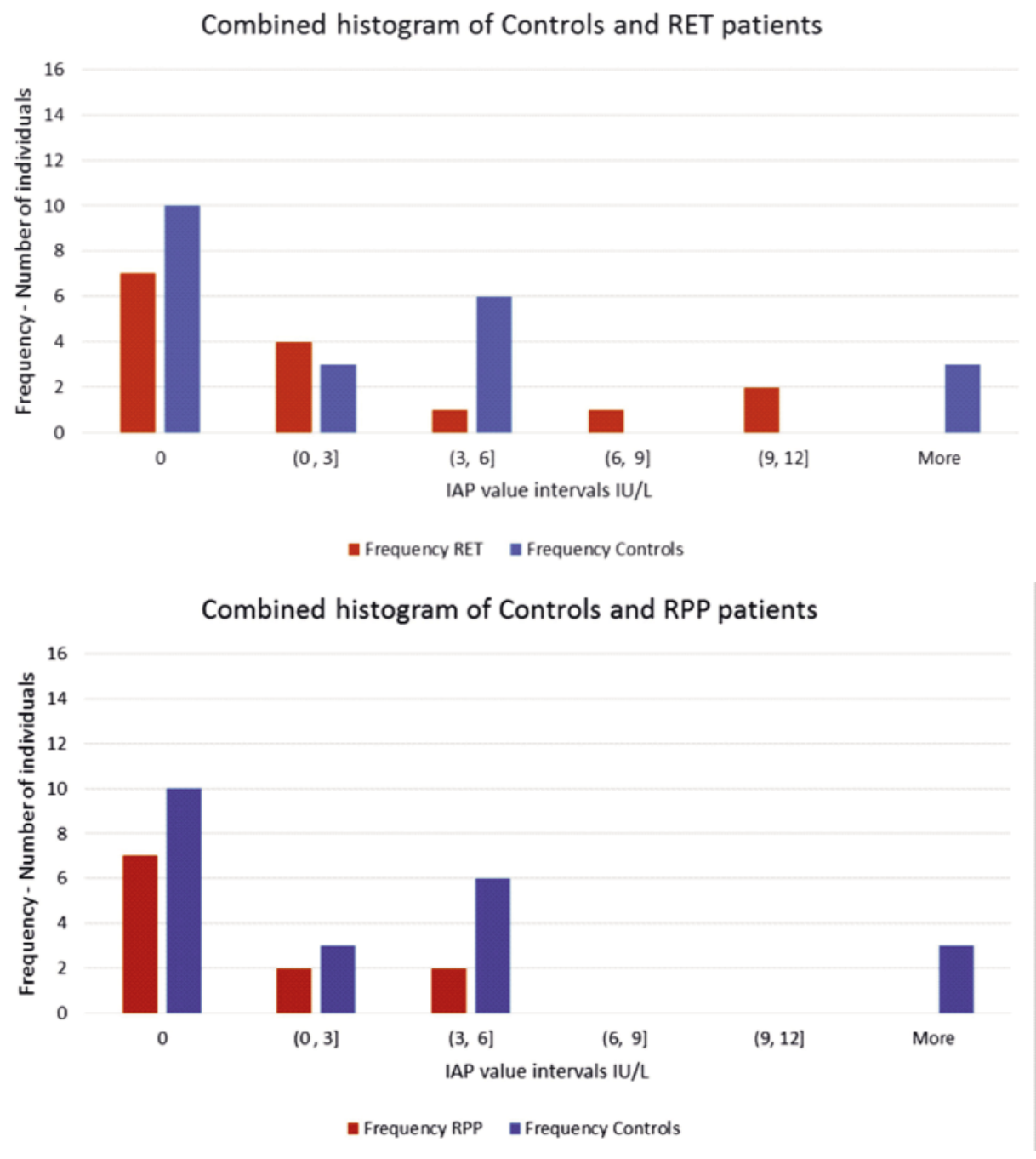

Figure 3 - Difference in IAP value distribution in erythematotelangiectatic (RET) and papulopustular rosacea (RPP) patients compared to control group 


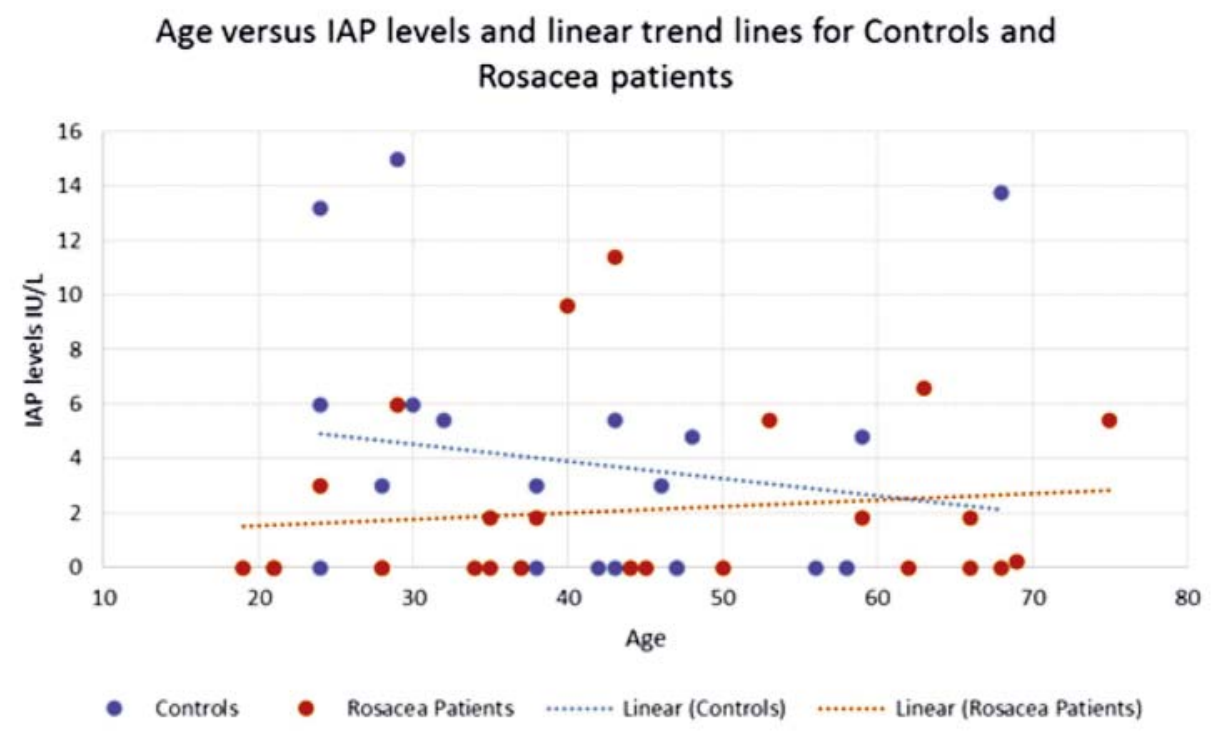

Figure 4 - Scatter plot of age versus IAP. Trend lines show that levels decrease with the age for controls and increase with the age for rosacea patients

\section{DISSCUSION}

The results of our study reveal that overall there is a slight but no statistically significant difference between IAP activity value in patients versus healthy controls. We observed that we have fewer individuals with no detectable IAP in our control group: 10 healthy individuals compared to 14 patients (6 with RET and 7 with RPP) had IAP enzymatic activity value $0.00 \mathrm{UI} / \mathrm{L}$. We also noticed fewer matched controls with small IAP values. In addition the highest normal values were distributed in the control group (13.17; 13.77; 14.97 IU/L). The subsample test that separated the rosacea patients according to their form of disease, indicated a significant difference in IAP mean enzymatic activity levels between patients diagnosed with papulopustular rosacea and the control group.

These findings suggest that IAP might have reduced activity in patients with inflammatory papulopustular rosacea and might play a role in its pathogenesis.

On the other hand, IAP values are significantly lower in those under 40 years of age, within the patient group

Combined histogram of Controls and Rosacea patients younger than 40

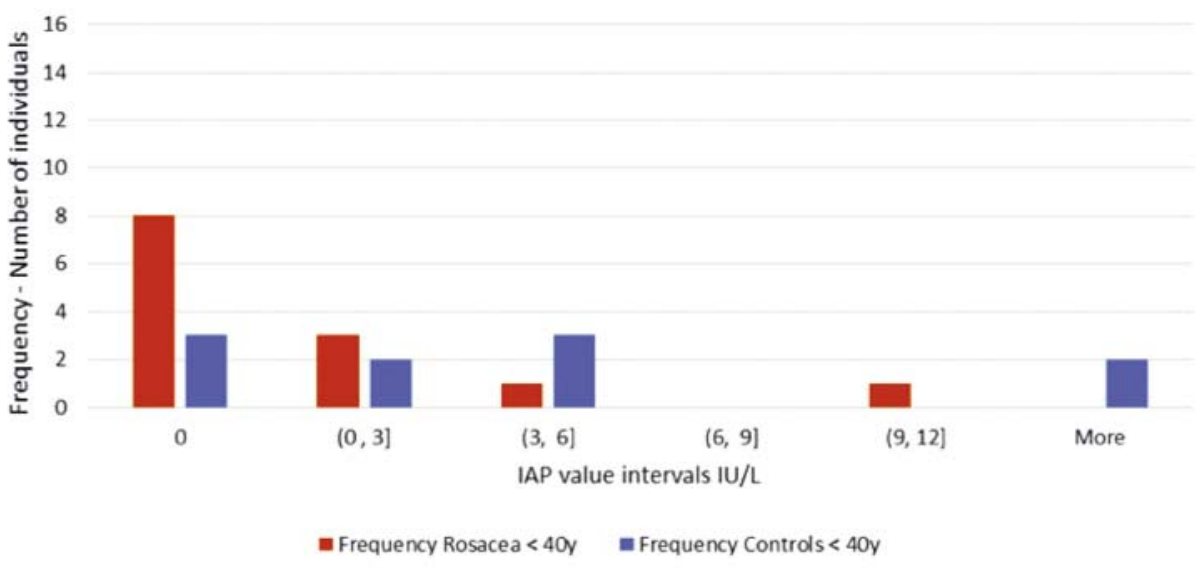

Figure 5 - IAP according to age threshold value of 40 years is statistically significantly lower in rosacea patients than matched controls 
compared to IAP values within the control group. In these patients IAP activity is lower and taking into account the fact that in adult population IAP activity does not differ between age groups (15), it supports the idea that IAP is important in rosacea evolution inyoung adults. A possible explanation might be that young people nowadays have a more chaotic lifestyle with an irregular and unbalanced diet, based onhighly processed foods and low in fresh vegetables, inducing an acidic $\mathrm{pH}$ in the intestine which inhibits IAP function. This coupled with a genetic predisposition to rosacea, which now we know it is real (16), might promote rosacea onset. Further studies on larger study groups need to be conducted in order to confirm our results and to assess if IAP is involved in late onset rosacea.

\section{CONCLUSION}

It appears that IAP might have a certain degree of influence in rosacea, especially in severe cases andin young adults in whom our study indicates that this enzyme has low activity. There might be also importance in studying if any therapeutically valuable result would be obtained in IAP supplementation in a subset of rosacea patients. So far, IAP administered intravenously has been successful in adjuvant therapy of necrotizing enterocolitis in neonates and sepsis in adults, by attenuation of the systemic inflammatory response and administered orally in moderate to severe ulcerative colitis, which led to short term improvement of the diseases (17-19). Patients with RPP could modify their diet, including more alkalizing foods in order to stimulate IAP enzyme function. A healthy IAP activity will prevent intestinal hormones or peptides from entering the bloodflow with its consequent increase in local cutaneous inflammation and vasodilation. We could debate that by mitigating inflammation in people affected by rosacea, administered IAP would be beneficial for these patients, but this hypothesis needs to be further evaluated. In addition larger study groups might be useful to definitely establish if IAP plays or not a main role in rosacea development, regardless of age.

\section{REFERENCES}

1. Chosidow 0, Cribier B. Epidemiology of rosacea: updated data. Ann Dermatol Venereol. 2011 Nov;138 Suppl 3:S179-83. doi: 10.1016/ S0151-9638(11)70087-4.
2. Wilkin J, Dahl M, Detmar M, Drake L, Liang MH, Odom R, et al. National Rosacea Society Expert Committee. Standard grading system for rosacea: report of the National Rosacea Society Expert Committee on the classification and staging of rosacea. J Am Acad Dermatol. 2004 Jun;50(6):907-12.

3. Wilkin J, Dahl M, Detmar M, Drake L, Feinstein A, Odom R, et al. Standard classification of rosacea: Report of the National Rosacea Society Expert Committee on the Classification and Staging of Rosacea. J Am Acad Dermatol. 2002 Apr;46(4):584-7.

4. Scharschmidt TC, Yost JM, Truong SV, Steinhoff M, Wang KC, Berger TG. Neurogenic rosacea: a distinct clinical subtype requiring a modified approach to treatment. Arch Dermatol. 2011 Jan;147(1):123-6. doi: 10.1001/archdermatol.2010.413.

5. Cresce ND, Davis SA, Huang WW, Feldman SR. The quality of life impact of acne and rosacea compared to other major medical conditions. J Drugs Dermatol. 2014 Jun;13(6):692-7.

6. Two AM, Wu W, Gallo RL, Hata TR. Rosacea: part I. Introduction, categorization, histology, pathogenesis, and risk factors. J Am Acad Dermatol. 2015 May;72(5):749-58; quiz 759-60. doi: 10.1016/j.jaad. 2014.08.028.

7. Holmes AD. Potential role of microorganisms in the pathogenesis of rosacea. J Am Acad Dermatol. 2013 Dec;69(6):1025-32. doi: 10.1016/ j.jaad.2013.08.006. Epub 2013 Sep 5.

8. Lazaridou E, Giannopoulou C, Fotiadou C, Vakirlis E, Trigoni A, loannides $D$. The potential role of microorganisms in the development of rosacea. J Dtsch Dermatol Ges. 2011 Jan;9(1):21-5. doi: 10.1111/ j.1610-0387.2010.07513.x. Epub 2010 Nov 8. English, German

9. De Hertogh G, Geboes K. Crohn's disease and infections: a complex relationship. Med Gen Med. 2004 Aug 10;6(3):14.

10. Merticariu A, Marinescu L, Giurc neanu C. Rosacea and its comorbidities J. Transl. Med. Res 2016;21(1):17-23. doi: 10.21614/ jtmr-21-1-68.

11. Whitehead J. Intestinal alkaline phosphatase: the molecular link between rosacea and gastrointestinal disease? Med Hypotheses. 2009 Dec;73(6):1019-22. doi: 10.1016/..mehy.2009.02.049. Epub 2009 Jul 1.

12. Estaki M, DeCoffe D, Gibson DL. Interplay between intestinal alkaline phosphatase, diet, gut microbes and immunity. World J Gastroenterol. 2014 Nov 14;20(42):15650-6. doi: 10.3748/ wjg.v20.i42.15650.

13. Laborator Synevo. Referinte specifice tehnologiei de lucru utilizate 2010. Ref Type: Catalog.

14. Wolff K, Johnson R, Saavedra A. Fitzpatrick's Color Atlas and Synopsis of Clinical Dermatology, 7th Edition, part 1, section 1. McGraw-Hill Education/Medical; 2013. P. 9-13.

15. Van Hoof VO, Hoylaerts MF, Geryl H, Van Mullem M, Lepoutre LG, De Broe ME. Age and sex distribution of alkaline phosphatase isoenzymes by agarose electrophoresis. Clin Chem. 1990 Jun;36(6):875-8.

16. Chang AL, Raber I, Xu J, Li R, Spitale R, Chen J, et al. Assessment of the genetic basis of rosacea by genome-wide association study. J Invest Dermatol. 2015 Jun;135(6):1548-55. doi: 10.1038/jid.2015.53. Epub 2015 Feb 19.

17. Riggle KM, Rentea RM, Welak SR, Pritchard KA, Oldham KT, Gourlay DM. Intestinal alkaline phosphatase prevents the systemic inflammatory response associated with necrotizing enterocolitis. J Surg Res. 2013 Mar;180(1):21-6. doi: 10.1016/j.jss.2012.10.042. Epub 2012 Nov 8.

18. Pickkers P, Heemskerk S, Schouten J, Laterre PF, Vincent JL, Beishuizen A, Jorens et al. Alkaline phosphatase for treatment of sepsis-induced acute kidney injury: a prospective randomized doubleblind placebo-controlled trial. Crit Care. 2012 Jan 23;16(1):R14. doi: 10.1186/cc11159.

19. Lukas M, Drastich P, Konecny M, Gionchetti P, Urban O, Cantoni F, et al. Exogenous alkaline phosphatase for the treatment of patients with moderate to severe ulcerative colitis. Inflamm Bowel Dis. 2010 Jul;16(7):1180-6. doi: 10.1002/ibd.21161. 